\title{
Decreasing Pancreatic Leak after Distal Pancreatectomy: Bovine Pericardium Wrapping Stump in a Porcine Model
}

\author{
Xin-yu Huang ${ }^{\mathrm{a}}$ Chen Wang $^{\mathrm{a}}$ Yong-zhi Liu $^{\mathrm{a}}$ Jia-zhe Liu ${ }^{\mathrm{a}}$ Hong-cheng Wang ${ }^{\mathrm{a}}$ \\ Qiong Jiao ${ }^{\mathrm{b}}$ Zhou Yuan $^{\mathrm{a}}{\text { Kai-xing } \mathrm{Al}^{\mathrm{a}}{ }^{\mathrm{Q}} \text { Qi Zheng }}^{\mathrm{a}}$ \\ Departments of a General Surgery, and ${ }^{b}$ Pathology, The Sixth People's Hospital of Shanghai Jiaotong University, \\ Shanghai, China
}

\section{Key Words}

Distal pancreatectomy · Pancreatic fistula - Bovine pericardium

\section{Abstract}

Background: Despite marked improvements in pancreatic surgery, the high incidence and morbidity of pancreatic leak after resection has remained unchanged. The current study investigated the safety and efficacy of bovine pericardium wrapping stump after distal pancreatectomy in a porcine model. Methods: Thirty-two swine were randomly assigned to control and experiment groups to undergo conventional scalpel transection with single hand-sewn closure of the pancreatic remnant (control) or bovine pericardium wrapping stump combined with hand-sewn closure (experiment). Closed-suction drainage was collected and measured daily. Animals were necropsied at 3 weeks postoperatively, and the pancreatic remnants were examined for histology. Primary end points were the development of a pancreatic fistula defined as greater than threefold drain/serum amylase after the 3rd postoperative day, and the presence of undrained amylase-rich fluid collections/abscess. Results: The incidence of pancreatic leak in the wrapping group was 6.3 versus $46.7 \%$ in the control group $(p<0.05)$. The amount of drainage fluid was higher in the control group than the experiment group during the postoperative days. There were no differences in operative time or other clinical parameters measured. No other significant differences were found in macroscopic changes between groups at reexploration. Histological examination demonstrated focal, chronic inflammation with necrosis in all animals. Conclusions: Bovine pericardium wrapping stump effectively reduced the incidence of pancreatic leakage after the distal pancreatectomy.

Copyright $\odot 2012$ S. Karger AG, Basel

\section{Introduction}

Distal pancreatectomy (DP) is the procedure of choice for benign or malignant lesions in the pancreatic body or tail. Despite a large variety of techniques for closure of the pancreatic stump after DP, pancreatic fistulas continue to

X.H. and C.W. contributed equally to this article.

\section{KARGER}

Fax +4161306 1234

E-Mail karger@karger.com

www.karger.com
(C) 2012 S. Karger AG, Basel

0253-4886/12/0296-0457\$38.00/0

Accessible online at:

www.karger.com/dsu
Xin-yu Huang

Department of General Surgery

The Sixth People's Hospital of Shanghai Jiaotong University

Shanghai (China)

E-Mail huangxinyush@hotmail.com 
be the most frequent and relevant surgical complication in this setting. At experienced high-volume centers, mortality after DP is currently below $5 \%$. However, there is considerable postoperative morbidity, around $20-40 \%$ [1-4]. Development of pancreatic fistulas increases the length of hospital stay and the cost of treatment, and can cause life-threatening complications such as abdominal bleeding, abscess formation, septicemia and so on. Therefore, it is essential to explore a safe and effective way to prevent pancreatic leakage. However, the coexistence of multiple technologies reflects the present situation that an effective and widely accepted stump-treating method is lacking in DP.

At present, the two best established methods for pancreatic stump closure are the suture technique after scalpel transection or only stapler technique. Hand suturing seems to be the preferred closure technique for the pancreatic remnant at various centers; it is defined as the current gold standard against which the safety of any new technique would have to be assessed [5]. However, there are many defects in the hand suturing technique, for example the sutures may cause tears within the pancreatic parenchyma and increase pancreatic leaks. In search of a better suturing technique, we experimented with the bovine pericardium wrapping stump before hand suturing, improving the hooping strength of pancreatic tissues, and avoiding cutting them. Based on that, we evaluated the efficacy of this material and technique in pancreatic surgery in a porcine model.

\section{Materials and Methods}

\section{Animals}

Thirty-two domestic swine (weighing 32.3-47.0 kg, mean 39.3 $\mathrm{kg}$ ) were used for the experiments in this study. All parts of this study were performed as part of an animal research protocol according to institution guidelines and reviewed and approved by the Institutional Animal Care and Use Committee, the Sixth People's Hospital of Shanghai Jiaotong University. Preoperative and anesthesia care was provided by fully trained veterinary staff members. Random allocation of animals to the single hand suture or bovine pericardium wrapping stump group was performed prior to anesthesia and surgical intervention.

\section{Anesthesia and Operative Technique}

Animals were fasted overnight prior to the experiments, but were allowed free access to water. We used ketamine ( $10 \mathrm{mg} / \mathrm{kg}$ i.m.) for initial intramuscular sedation, and intravenous access was obtained by way of marginal ear vein cannulation using large gauge venous catheters. Atropine $(0.05 \mathrm{mg} / \mathrm{kg}$ i.v. $)$ was given $5 \mathrm{~min}$ before intubation to help dry secretions, and anesthesia was maintained by $1 \%$ napental $(0.5 \mathrm{ml} / \mathrm{kg}$ i.v. $)$ intravenously as needed. To ensure free airways and to be prepared in case of an emergency, the pigs were intubated endotracheally. Perioperative fluids consisted of Plasmalyte (balanced salt solution) at $20 \mathrm{ml} / \mathrm{kg}$. Oxygen saturation and heart rate were monitored using pulse oximetry placed on the ear.

After the anesthesia, the abdomen was prepared with povidone-iodine, and was draped in sterile fashion. A midline laparotomy using scalpel and electrosurgical dissection was used for intraabdominal access extending from the xiphoid to the umbilicus to gain access to the peritoneal cavity. The intraperitoneally located pancreatic tail was identified and freed from adjacent jejunum and surrounding tissue until about $7-8 \mathrm{~cm}$ of the distal pancreas were freely movable (fig. 1a). Typical for pigs, the spleen and splenic vein do not adhere tightly to this part of the pancreas and could be preserved. In the conventional single resection group, the pancreas was merely oversewn using 3-0 PROLENE sutures (Provided by ETHICON Inc.) at the middle and outward $1 / 3$ points, which was about $0.3-0.5 \mathrm{~cm}$ from the pancreatic stump; then, the two threads were fixed on the opposite side of the pancreas, and the three stay sutures were tied respectively; finally, the $3 \mathrm{U}$ type lock-stitch sutures were made on each $1 / 3$ tissue cross-section. Hemostasis was obtained with fine suture ligatures (fig. 1b). Approximately $5 \mathrm{~cm}$ of the distal pancreas was then transected by scalpel, and the specimen removed. In the experiment group, the distal pancreas was looped by bovine pericardium (Cingular Biotech Corporation, Shanghai, China), making sure the rough side was towards the pancreas and the smooth side towards the intestine. Next, we used the same sutures passing through the wrapped pancreas, and then we ligatured them the same way as in the control group. By this means, the stump of the pancreas was tightly closed and the rupture of pancreatic tissue was avoided as much as possible (fig. 1c, d). In all animals, the pancreatic duct was too small to be located at the transection line. After stump management, a closed-suction drain (Provided by Huaxia Medical Supplies Institute of Shanghai, China.) was placed with its tip located adjacent to the pancreatic stump and the proximal end tunneled subcutaneously to the dorsum of the animal and connected to a collection chamber. The fascia was then closed with 0 MERSILK in a running fashion, and the skin and subcutaneous tissue closed with 4-0 MERSILK subcuticular running sutures.

\section{Postoperative Procedures}

After the procedure, the animals were recovered in a monitored setting for several hours and then extubated when clinically indicated. Once ambulating, they were housed in individual large animal facilities at our institution. Prophylactic doses of antibiotics (penicillin, 2.4 million $\mathrm{U}$ i.m.) were administered for the first 3 postoperative days. All animals received sauteralgyl (100 mg i.m.) for postoperative analgesia on the first 2 postoperative days, and then on an as needed basis. All animals were given ad libitum water only for the first $24 \mathrm{~h}$ and subsequently fed twice daily with pig chow.

\section{Data Collection}

Peripheral blood was collected prior to pancreatic resection and just before sacrifice for measurement of systemic pancreatic enzyme levels. All animals were inspected twice daily by both the veterinary team and the authors in a blinded fashion to assess for fever, food intake, lethargy, emesis, anorexia, and bowel function in an effort to identify any suspicious clinical signs of complications. Closed-suction drainage output and quality were quantified 

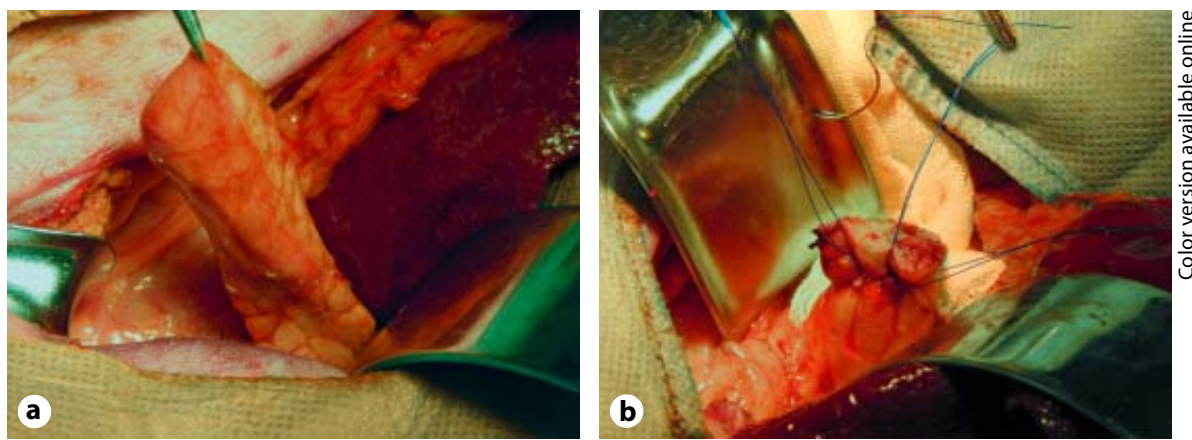

Fig. 1. a Distal pancreas was prepared. b Remnant was oversewn in the control after traditional hand suturing. In the experiment group, stump was wrapped in bovine pericardium (c), and remnant was oversewn after wrapping $(\mathbf{d})$.
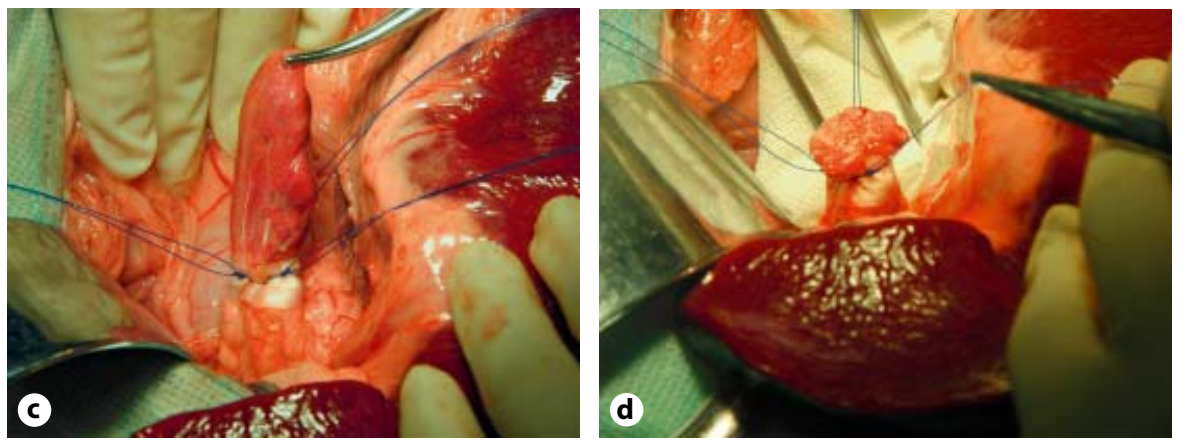

daily for the first 10 postoperative days, and amylase was determined at the conclusion of the study. Drainage fluid samples were analyzed semiautomatically at the hospital's centralized analysis center by technicians who were unaware of the study groups.

\section{Necropsy}

All the animals in each group underwent necropsy at 3 weeks after the initial procedure to account for any late complications. They were all killed with a commercial euthanasia solution. Intraabdominal pathologic findings (e.g. fluid collection, abscesses) were recorded, and a portion of the pancreas including the resection margin was collected in formalin for histologic evaluation. Paraffin sections were stained with hematoxylin and eosin. Histologic evaluation was performed by a pathologist in a blinded fashion.

\section{Clinical Observation}

The primary outcomes studied in our experiment were the development of a postoperative pancreatic leak defined as greater than threefold drain/serum amylase after the 3rd postoperative day (as defined by the ISGPF) [6], surgical complications, and any undrained amylase-rich fluid collections/abscess. Secondary outcomes were operative time, wound infection, total daily drain output, and other perioperative clinical parameters (anorexia, emesis, and lethargy).

\section{Statistical Analysis}

Data are presented as mean \pm standard error of the mean for continuous variables. Categorical variables were compared between groups using Fisher's exact test. Comparisons between groups for continuous variables were carried out using Student's t tests. SPSS version 13.0 (SPSS, Chicago, Ill., USA) was used for all analyses. Statistical significance was accepted at $\mathrm{p}<0.05$.

Bovine Pericardium Wrapping Stump in Distal Pancreatectomy

\section{Results}

There were no deviations from the protocol as described in the methodology. There was one intraoperative death (control group) because of anesthesia recovery failure; otherwise, all animals survived the intraoperative procedures. A total of 31 animals (15 control, 16 experiment) were included in the study.

As described previously, systemic baseline levels of amylase are much higher in pigs than in humans [7], and were around 2,000 $\mathrm{U} / \mathrm{l}$. When drainage/serum ratios of amylase were calculated, the total incidence of pancreatic leak in the experiment group was 6.2 versus $46.7 \%$ in the control group ( $\mathrm{p}<0.05$; fig. 2). The amount of drainage fluid was higher in the control group than in the experiment group at day 2 ( $\mathrm{p}<0.01$; fig. 3 ).

The range of operative time was $85-125 \mathrm{~min}$ and did not differ significantly between groups (control $102 \pm$ $11.2 \mathrm{~min}$, wrapping $107 \pm 11.1 \mathrm{~min} ; \mathrm{p}=0.31$ ). There were also no differences in animal bodyweight, and no differences in clinical parameters such as fever, lethargy or anorexia between groups (table 1). When animals were sacrificed at 3 weeks after operation, small fluid collections/pseudocysts or abscesses were infrequently found at abdominal reexploration (3 control, 1 wrapping); there was no significant abnormality around the bovine pericardium in the wrapping pancreatic stump (fig. 4); 4 ani- 


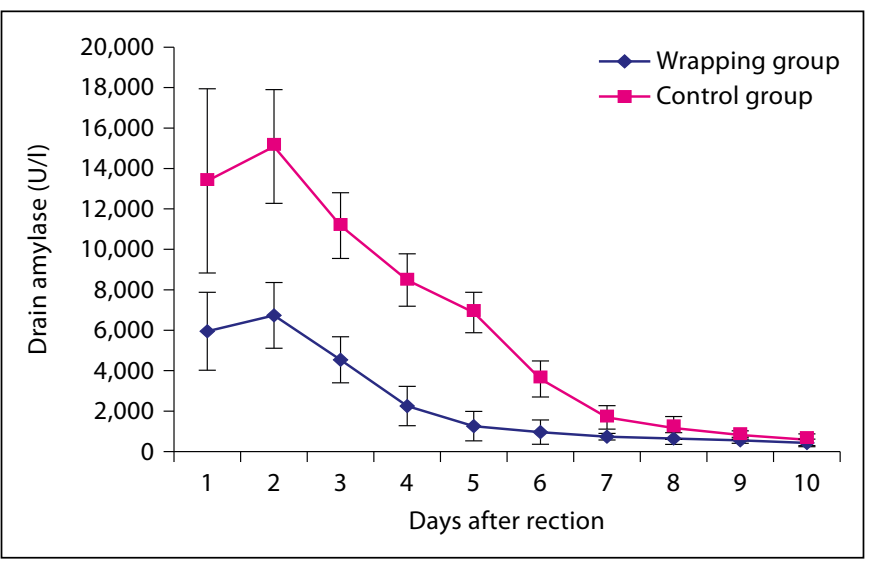

Fig. 2. Total average drain amylase activity in the control and wrapping groups over time. The rate of pancreatic leak in the wrapping group was 6.2 vs. $46.7 \%$ in the control group $(\mathrm{p}<0.05)$.

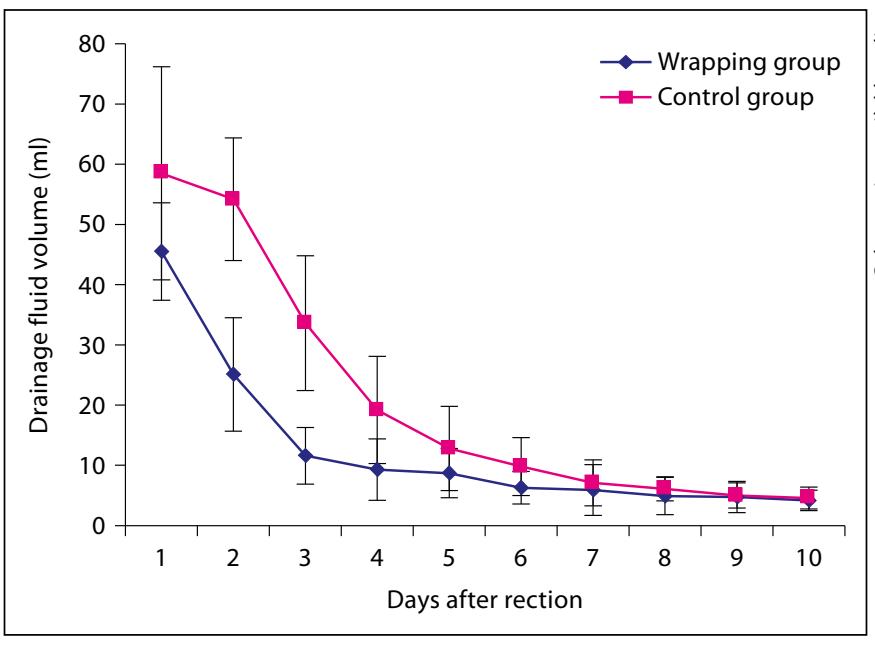

Fig. 3. The amount of drainage fluid was higher in controls than in the wrapping group. At postoperative day 2 , it was significantly higher in controls compared to the wrapping group $(\mathrm{p}<0.01)$.

Table 1. Various other parameters measured in the control and wrapping animals

\begin{tabular}{lccl}
\hline Variable & Wrapping & Control & p value \\
\hline Bodyweight, kg & $39.9 \pm 3.4$ & $38.7 \pm 3.9$ & 0.33 \\
Operative time, min & $107 \pm 11.1$ & $102 \pm 11.2$ & 0.31 \\
Outcomes & & & \\
$\quad$ Fever & 1 & 5 & 0.22 \\
$\quad$ Lethargy & 1 & 1 & 1.00 \\
$\quad$ Anorexia & 1 & 2 & 0.60 \\
$\quad$ Wound infection & 3 & 4 & 0.69 \\
$\quad$ Fluid collections/pseudocysts & 1 & 3 & 0.33 \\
\hline
\end{tabular}

mals in the control group and 3 animals in the wrapping group had evidence of wound infection, but this did not reach statistical significance.

Histopathological evaluation demonstrated focal inflammation with focal necrosis at the resection margin in the wrapping group. Furthermore, control group animals had evidence of pyogranulomatous inflammation with necrosis around the reaction and fibrous scar formation surrounding the normal pancreas (fig. 5).

\section{Discussion}

A durable closure of the pancreatic remnant is the Achilles' heel in DP. Formation of a pancreatic fistula that is potentially associated with further complications (e.g. wound infection, intraabdominal abscess, hemorrhage, sepsis) is the main cause of surgical morbidity [1]. Leaks after DP increase the cost of care and subsequent health care resource utilization. Currently, most scholars believe that sutures cutting through the soft texture of the pancreas and the failure of main pancreatic duct ligation are the risk factors of pancreatic leakage in the DP [8-11]. Numerous closure techniques of the pancreatic remnant have been introduced in an attempt to reduce surgical morbidity, such as the hand-sewn sutures, stapled closure, pancreatic-intestinal anastomosis, pancreatic-gastric anastomosis, fibrin glue sealing method, saline-coupled radiofrequency ablation, LigaSure, gastric serosal patch, jejunal seromuscular flap, falciform ligament pedicle flap, and the combination of those methods, etc. [1220]. However, the optimal surgical procedure has yet to be defined. Currently, hand suturing seems to be the preferred closure technique for the pancreatic remnant at various centers; it is defined as the 'gold standard against which the safety of any new technique would have to be assessed [5].

It has been proposed that a soft, friable, and normal pancreas of normal size with a thin-walled main pancreatic duct increases the risk of pancreatic fistula. In the process of hand suturing, if knot strength is excessive, the pancreas can be easily cut and result in pancreatic leakage; with little stress, the ligation may be insufficient during the suturing or after operation. Traditional oversewing of the gland with sutures does not completely close the stump leading to persistent extravasation of enzyme-rich pancreatic fluid, which leads to subsequent duct disruption and leak. Furthermore, the sutures themselves may cause tears within the pancreatic parenchyma and increase pancreatic leaks. In the 
Fig. 4. a Abscesses with necrosis were found in the pancreatic stump 3 weeks after surgery in the control group (arrow points to necrosis). b There was no significant abnormality in the pancreatic stump in the wrapping group (arrow points to bovine pericardium).

Fig. 5. a Wrapping at 3 weeks. The bovine pericardium was collagen tissue with little macrophages infiltrating (big arrows). Some evidence of hemorrhage and necrosis with focal inflammation (small arrows) was found between the stump and biomembrane, but no foreign body granuloma. b Control at 3 weeks. Lots of hemorrhagic and necrotic tissues were found around the pancreatic stump (small arrows), and some fibrous connective tissues surrounded the normal pancreas (big arrow). HE. $\times 40$.
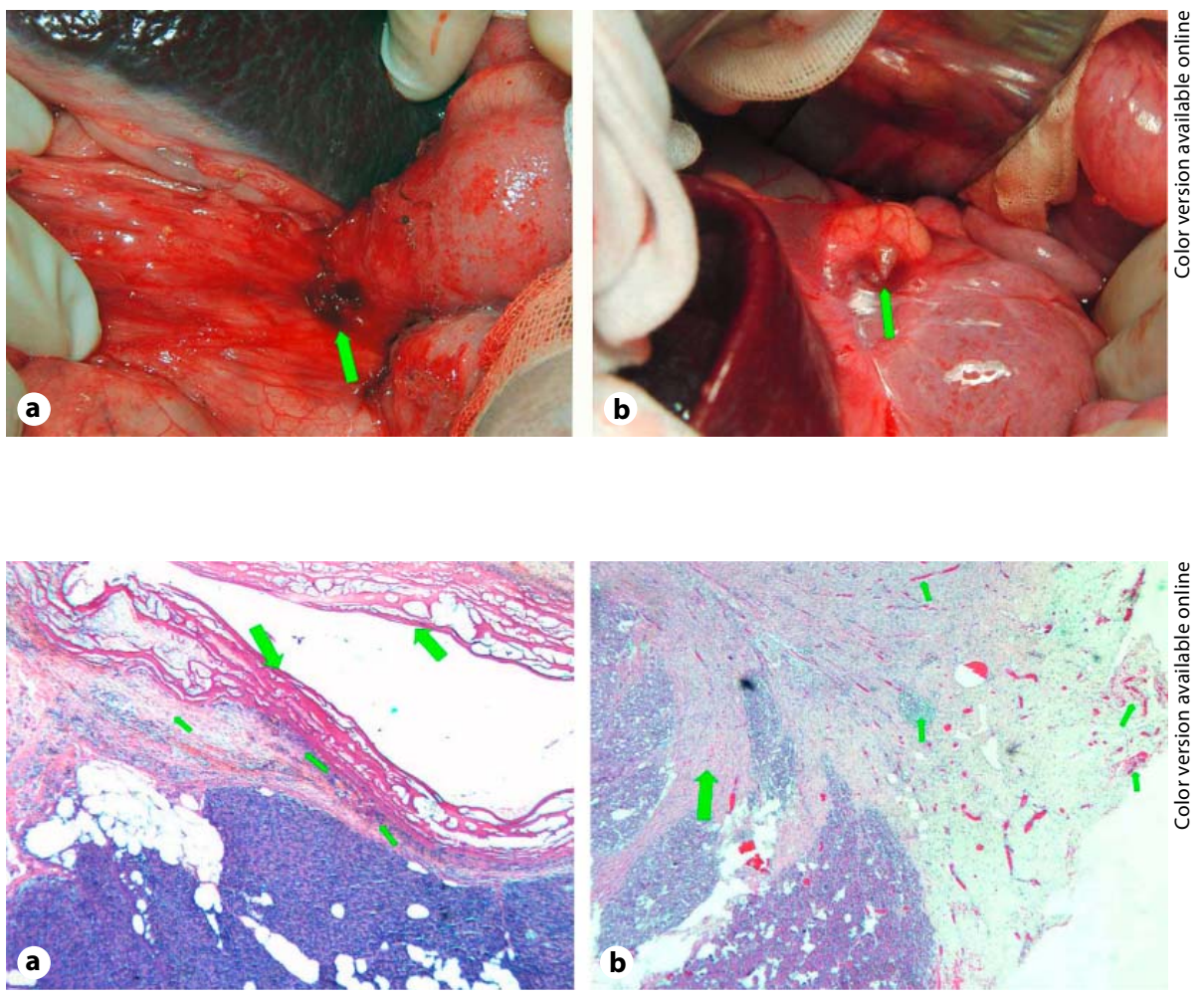

present study, we compared the bovine pericardium wrapping stump and the single hand suture technique for closure of the pancreatic remnant in a porcine model of DP. In the experiment group, when the stump was wrapped, the pancreatic parenchyma was able to withstand the suturing because of good compactness. The cutting caused by sutures was also limited, and as the cut resistance of the pancreatic tissue was significantly enhanced, the suture loop tightness could be achieved as safely as possible. Our data show that the pancreas stump could be safely closed by the bovine pericardium. Only one $(6.3 \%)$ postoperative pancreatic fistula was found in the group with wrapped stump, whereas seven were seen $(46.7 \%)$ in the single hand-sewn closure group. Moreover, the amount of drainage fluid was lower in the wrapping group than in the conventional resection group because suture loop tightness was enhanced. No significant decomposition or absorption of bovine pericardium 3 weeks after operation was observed, and no significant differences between the two groups were found regarding other relevant parameters, including abscesses, fluid collections, or laboratory-defined pancreatitis. Based on our experiments, we believe bovine pericardium wrapping stump is a safe stump closure technique.

Bovine Pericardium Wrapping Stump in Distal Pancreatectomy
Bovine pericardium has been widely used in cardiac surgery and dura mater transplants [21-22]. This material has been deconstructed as biocompatible and does not cause immune response. The bovine pericardium has a rough and a smooth side. We put the rough side towards the pancreas and the smooth side towards the bowel. The rough side promotes adhesion with the pancreas tissue, the smooth side has an anti-adhesive property, reducing adhesion with the bowel, and reducing the incidence of adhesive intestinal obstruction significantly. Complications of the fibrin glue or patch or flap techniques such as intestinal obstruction, total necrosis of the flap, and infection have been reported frequently [23-24].

In our study, the high amylase levels and drainage fluid levels did not contribute to severe clinical outcomes such as abscess formation and septicemia. Good operating skills and perioperative management were very important, and all animals which developed pancreatic fistula had a temporary grade A fistula (as defined by the ISGPF). The overall level of enzyme-rich pancreatic fluid at the resection bed was limited because of adequate drainage; therefore, no severe complications were observed in the two groups. The high amylase level and high drainage fluid level were observed only in the early postoperative period, and were similar from postoperative 
day 7. The experiment group had mean drain amylase concentrations far below the current definition of a biochemical leak. In contrast, the swine in the control group had levels far in excess of this defining cutoff. The clinical consequences of this perhaps would have been apparent if we had a larger sample size. Further studies would be necessary to support this hypothesis.

There are some important technical considerations to keep in mind when using this material for pancreatic resection surgery. Concerning the biomaterial, we should pay attention to the durability problem at present, mainly related to absorption and degradation. Further controlled randomized studies involving large numbers of patients are warranted to confirm the value of the present surgical option.

\section{Conclusions}

It is evident that there is a need for improvement in the prevention of postoperative pancreatic leak after pancreatic resection. The current techniques and methodologies have been unsuccessful in minimizing the incidence of this complication which is a main cause of morbidity and increases financial costs of pancreatic resection. Bovine pericardium wrapping stump is a new modality that shows promise in limiting pancreatic leak after resection in our animal model. Its excellent applicability makes this wrapping technique very attractive for future studies in pancreatic surgery. Based on our results, human trials would be a reasonable and logical next step toward assessing the clinical utility of the biomembrane in pancreatic surgery.

\section{References}

$>1$ Ridolfini MP, Alfieri S, Gourgiotis S, Di $>_{10}$ Pannegeon V, Pessaux P, Sauvanet A, VulMiceli D, Rotondi F, Quero G, Manghi R, Doglietto GB: Risk factors associated with pancreatic fistula after distal pancreatectomy, which technique of pancreatic stump closure is more beneficial? World J Gastroenterol 2007;13:5096-5100.

$\checkmark 2$ Christein JD, Kendrick ML, Iqbal CW, Nagorney DM, Farnell MB: Distal pancreatectomy for respectable adenocarcinoma of the body and tail of the pancreas. Gastrointest Surg 2005;9:922-927.

3 Velanovich V: Case-control comparison of laparoscopic versus open distal pancreatectomy. J Gastrointest Surg 2006;10:95-98.

4 KnaebelHP, Diener MK, Wente MN, Büchler MW, Seiler CM: Systematic review and meta-analysis of technique for closure of the pancreatic remnant after distal pancreatectomy. Br J Surg 2005;92:539-546.

$\checkmark 5$ Diener MK, Seiler CM, Rossion I, et al: Efficacy of stapler versus hand-sewn closure after distal pancreatectomy (DISPACT): a randomised, controlled multicentre trial. Lancet 2011;377:1514-1522.

6 Bassi C, Dervenis C, Butturini G, et al: Postoperative pancreatic fistula: an international study group (ISGPF) definition. Surgery 2005;138:8-13.

7 Kraft W, Dürr U (eds): Klinische Labordiagnostik in der Tiermedizin. Stuttgart, Schattauer, 1995, pp 122-123.

$>8$ Farkas G, Leindler L, Farkas GJR: Safe closure technique for distal pancreatic resection. Langenbecks Arch Surg 2005;390:29-31.

$>9$ Rodríguez JR, Germes SS, Pandharipande PV, Gazelle GS, Thayer SP, Warshaw AL, Fernández-del Castillo C: Implications and cost of pancreatic leak following distal pancreatic resection. Arch Surg 2006;141:361-366. lierme MP, Kianmanesh R, Belghiti J: Pancreatic fistula after distal pancreatectomy: predictive risk factors and value of conservative treatment. Arch Surg 2006;141:10711076.

$>11$ Reeh M, Nentwich MF, Bogoevski D, Koenig AM, Gebauer F, Tachezy M, Izbicki JR, Bockhorn M: High surgical morbidity following distal pancreatectomy: still an unsolved problem. World J Surg 2011;35:1110-1117.

12 Wagner M, Gloor B, Ambühl M, Worni M, Lutz JA, Angst E, Candinas D: Roux-en-Y drainage of the pancreatic stump decreases pancreatic fistula after distal pancreatic resection. J Gastrointest Surg 2007;11:303308.

13 Ikegami T, Maeda T, Kayashima H, Oki E, Yoshizumi T, Sakaguchi Y, Toh Y, Shirabe K, Maehara Y: Soft coagulation, polyglycolic acid felt, and fibrin glue for prevention of pancreatic fistula after distal pancreatectomy. Surg Today 2011;41:1224-1227.

14 Suc B, Msika S, Fingerhut A, Fourtanier G, et al: Temporary fibrin glue occlusion of the main pancreatic duct in the prevention of Intra-abdominal complications after pancreatic resection. Ann Surg 2003;237:57-65.

15 Truty MJ, Sawyer MD, Que FG: Decreasing pancreatic leak after distal pancreatectomy: saline-coupled radiofrequency ablation in a porcine model. J Gastrointest Surg 2007;11: 998-1007.

16 Hartwig W, Duckheim M, Strobel O, Dovzhanskiy D, Bergmann F, Hackert T, Büchler MW, Werner J: LigaSure for pancreatic sealing during distal pancreatectomy. World J Surg 2010;34:1066-1070.
17 Kluger Y, Alfici R, Abbley B, Soffer D, Aladgem D: Gastric serosal patch in distal pancreatectomy for injury: a neglected technique. Injury 1997;28:127-129.

18 Walters DM, Stokes JB, Adams RB, Bauer TW: Use of a falciform ligament pedicle flap to decrease pancreatic fistula after distal pancreatectomy. Pancreas 2011;40:595-599.

19 Issekutz A, Belágyi T, Romics L Jr, Oláh A: A novel technique for the closure of the pancreatic remnant using jejunal serosa following distal pancreatectomy. Magy Seb 2006;59: 117-121.

20 Sudo T, Murakami Y, Uemura K, Hayashidani Y, Hashimoto Y, Nakashima A, Ohge $H$, Sueda T: Distal pancreatectomy with duct-to-mucosa pancreaticogastrostomy: a novel technique for preventing postoperative pancreatic fistula. Am J Surg 2011;202: 77-81.

21 Li X, Guo Y, Ziegler KR, Model LS, Eghbalieh SD, Brenes RA, Kim ST, Shu C, Dardik A: Current usage and future directions for the bovine pericardial patch. Ann Vasc Surg 2011;25:561-568.

22 Baharuddin A, Go BT, Firdaus MN, Abdullah J: Bovine pericardium for dural graft: clinical results in 22 patients. Clin Neurol Neurosurg 2002;104:342-344.

23 Wei HJ, Liang HC, Lee MH, Huang YC, Chang Y, Sung HW: Construction of varying porous structures in acellular bovine pericardia as a tissue-engineering extracellular matrix. Biomaterials 2005;26:1905-1913.

24 Maeda A, Ebata T, Kanemoto H, Matsunaga $\mathrm{K}$, Bando E, Yamaguchi S, Uesaka K: Omental flap in pancreaticoduodenectomy for protection of splanchnic vessels. World J Surg 2005;29:1122-1126. 